\title{
Nivolumab Induces Sustained Liver Injury in a Patient with Malignant Melanoma
}

\author{
Tokuhiro Matsubara ${ }^{1}$, Tsutomu Nishida ${ }^{1}$, Yu Higaki ${ }^{1}$, Ryo Tomita ${ }^{1}$, Hiromi Shimakoshi ${ }^{1}$, \\ Akiyoshi Shimoda ${ }^{1}$, Naoto Osugi ${ }^{1}$, Aya Sugimoto ${ }^{1}$, Kei Takahashi ${ }^{1}$, Dai Nakamatsu ${ }^{1}$, \\ Kaori Mukai ${ }^{1}$, Masashi Yamamoto ${ }^{1}$, Koji Fukui ${ }^{1}$, Shiro Adachi ${ }^{2}$ and Masami Inada ${ }^{1}$
}

\begin{abstract}
:
A 42-year-old man was diagnosed with cStage IIIb malignant melanoma and underwent resection. After interferon-beta therapy, 18-fluorodeoxyglucose-positron emission tomography/computed tomography $\left({ }^{18} \mathrm{~F}\right.$ FDG PET/CT) showed multiple lung metastases, and he received nivolumab $(2 \mathrm{mg} / \mathrm{kg})$ every 3 weeks, resulting in a total of 17 cycles. After treatment, ${ }^{18} \mathrm{~F}$-FDG PET/CT showed a significant decrease in the size of the metastases, but he had a Grade 4 alanine aminotransferase (ALT) elevation. Liver histology revealed druginduced liver damage. Therefore, we performed steroid half-pulse therapy followed by oral methylprednisolone, but his ALT level did not completely recover to the normal range even after five months. We herein report a case with specific, sustained liver injury induced by nivolumab as an immune-related adverse events.
\end{abstract}

Key words: nivolumab, immune checkpoint inhibitors, immune-related adverse events, persistent liver injury

(Intern Med 57: 1789-1792, 2018)

(DOI: 10.2169/internalmedicine.9851-17)

\section{Introduction}

Immune checkpoint inhibitors have recently become widely used as new cancer therapeutic agents to enhance antitumor immunity (1). Nivolumab is a recombinant monoclonal immunoglobulin G4 to programmed cell death receptor 1 (PD-1) and leads to remarkable clinical responses in patients (2). However, the augmented immune response enabled by immune checkpoint inhibitors also leads to immune-related adverse events (irAEs) (3), and little is known regarding the details of these events, including hepatitis, which is reported to occur in $1.0-3.0 \%$ of nivolumabtreated patients (4). Most cases of hepatitis are mild to moderate in severity, and severe and sustained liver dysfunction is rare. We herein report a case of nivolumab-induced persistent liver injury in a patient with malignant melanoma.

\section{Case Report}

A 42-year-old man presented with a tumor of the left fe- mur. A biopsy revealed malignant melanoma. Positron emission tomography with 2-deoxy-2-[fluorine-18]fluoro-Dglucose integrated with computed tomography $\left({ }^{18} \mathrm{~F}-\mathrm{FDG}\right.$ PET/CT) showed an abnormal uptake of the primary tumor and left inguinal lymph node. The patient was diagnosed with malignant melanoma (cT4bN1bM0, cStage IIIb), and he underwent radical resection. He then received 11 cycles of interferon-beta at doses of 3 million international units as adjuvant therapy. After treatment, ${ }^{18} \mathrm{~F}-\mathrm{FDG}$ PET/CT showed multiple lung metastases. He was diagnosed with distant recurrence and received nivolumab $(2 \mathrm{mg} / \mathrm{kg})$ every 3 weeks from January 2016.

He developed Grade 3 serum alanine aminotransferase (ALT) elevation after the first cycle of nivolumab at week 3, but the liver function spontaneously recovered. He ultimately received a total of 17 cycles of nivolumab over 1 year. ${ }^{18}$ F-FDG PET/CT showed a significant decrease in the ${ }^{18} \mathrm{~F}$-FDG uptake of the lung metastatic lesions, but recurrent Grade 4 ALT elevation was noted after 17 cycles of nivolumab at week 3 , so he was admitted to our hospital.

He had no specific physical findings, but his laboratory

${ }^{1}$ Department of Gastroenterology, Toyonaka Municipal Hospital, Japan and ${ }^{2}$ Department of Pathology, Toyonaka Municipal Hospital, Japan Received: July 20, 2017; Accepted: November 8, 2017; Advance Publication by J-STAGE: February 9, 2018

Correspondence to Dr. Tokuhiro Matsubara, tmatsubara@chp.toyonaka.osaka.jp 
studies showed elevation of the ALT level of $785 \mathrm{U} / \mathrm{L}$ with eosinophilia at $12.5 \%(1,138 / \mu \mathrm{L})$ but no significant biliary enzyme elevation. No other causes for the liver dysfunction than nivolumab, such as viral hepatitis, fatty liver, liver metastasis, autoimmune hepatitis or primary biliary cholangitis, were recognized (Table and Fig. 1). In addition, no other drugs had been administered around the same time as nivolumab.

Fig. 2 shows the time course of ALT, aspartate amino-

Table. Laboratory Data on Admission.

\begin{tabular}{|c|c|c|c|}
\hline \multicolumn{2}{|l|}{ Biochemical data } & \multirow{3}{*}{$\begin{array}{l}\mathrm{CRP}(\mathrm{mg} / \mathrm{dL}) \\
\mathrm{Na}(\mathrm{mEq} / \mathrm{L}) \\
\mathrm{K}(\mathrm{mEq} / \mathrm{L})\end{array}$} & \multirow{2}{*}{$\begin{array}{r}0.23 \\
139\end{array}$} \\
\hline $\mathrm{WBC}(/ \mu \mathrm{L})$ & 6,700 & & \\
\hline $\mathrm{RBC}\left(\times 10^{4} / \mu \mathrm{L}\right)$ & 509 & & 4.7 \\
\hline $\mathrm{Hb}(\mathrm{g} / \mathrm{dL})$ & 15.1 & \multicolumn{2}{|c|}{ Immunological and other data } \\
\hline Platelets $\left(\times 10^{4} / \mu \mathrm{L}\right)$ & 23.5 & $\mathrm{IgG}(\mathrm{mg} / \mathrm{dL})$ & 880 \\
\hline $\mathrm{PT}(\%)$ & 92 & $\operatorname{IgM}(\mathrm{mg} / \mathrm{dL})$ & 44 \\
\hline PT-INR & 1.04 & $\operatorname{IgA}(\mathrm{mg} / \mathrm{dL})$ & 205 \\
\hline D-dimer $(\mu \mathrm{g} / \mathrm{mL})$ & 0.2 & ANA & $<40$ \\
\hline $\operatorname{AST}(\mathrm{U} / \mathrm{L})$ & 254 & AMA & $<20$ \\
\hline $\operatorname{ALT}(\mathrm{U} / \mathrm{L})$ & 693 & AMA-M2 (Index) & $<1.5$ \\
\hline LDH (U/L) & 252 & $\mathrm{TSH}(\mu \mathrm{IU} / \mathrm{mL})$ & 2.7 \\
\hline $\operatorname{ALP}(\mathrm{U} / \mathrm{L})$ & 373 & Free T4 (ng/dL) & 1.28 \\
\hline 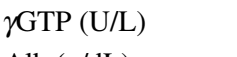 & 87 & Free T3 $(\mathrm{pg} / \mathrm{mL})$ & 2.42 \\
\hline $\operatorname{Alb}(\mathrm{g} / \mathrm{dL})$ & $\begin{array}{r}3.5 \\
0.61\end{array}$ & \multicolumn{2}{|l|}{ Viral markers } \\
\hline $\begin{array}{l}\text { T-Bil (mg/dL) } \\
\text { D-Bil (mg/dL) }\end{array}$ & $\begin{array}{l}0.61 \\
0.09\end{array}$ & HBsAg (IU/mL) & 0.0 \\
\hline BUN (mg/dL) & 22 & $\mathrm{HBcAb}(\mathrm{S} / \mathrm{CO})$ & 0.1 \\
\hline $\mathrm{Cr}(\mathrm{mg} / \mathrm{dL})$ & 0.68 & $\mathrm{HBsAb}(\mathrm{mIU} / \mathrm{mL})$ & 0 \\
\hline $\mathrm{UA}(\mathrm{mg} / \mathrm{dL})$ & 7.0 & HCVAb (S/CO) & 0.1 \\
\hline & & HAVAb (S/CO) & 0.65 \\
\hline
\end{tabular}

WBC: white blood cell, RBC: red blood cell, Hb: hemoglobin, PT: prothrombin time, AST: aspartate aminotransferase, ALT: alanine aminotransferase, LDH: lactate dehydrogenase, ALP: alkaline phosphatase, $\gamma$ GTP: gamma-glutamyltranspeptidase, Alb: albumin, T-Bil: total bilirubin, D-Bil: direct bilirubin, BUN: blood urea nitrogen, Cr: creatinine, UA: uric acid, CRP: C-reactive protein, Na: natrium, K: potassium, IgG/IgA/ IgM: immunoglobulin G/A/M, ANA: antinuclear antibody, AMA: antimitochondrial antibody, TSH: thyroid-stimulating hormone, T4: thyroxine, T3: triiodothyronine, HBsAg/Ab: hepatitis B surface antigen/antibody, HBcAb: hepatitis B core antibody, HCVAb: hepatitis C virus antibody, HAVAb: hepatitis A virus antibody transferase (AST), eosinocytes and gamma-glutamyltranspeptidase (GTP). A liver biopsy was performed to identify the cause of the abnormal liver enzyme levels. It showed scattered necrotic foci accompanied by a small number of macrophages, lymphocytes and eosinophils in the centrilobular zone but no cholestasis or plasma cell-rich mononuclear infiltration. The portal area was inflamed with a moderate number of inflammatory cells, including lymphocytes and eosinophils. Neither portal fibrosis nor bridging fibrosis was found (Fig. 3). Nivolumab was discontinued, and liver supporting therapy was performed starting at 12 days after hospitalization.

The liver enzymes decreased slightly, but the ALT level failed to fully recover. We considered this to be an irAE. We started the patient on oral methylprednisolone (mPSL) (0.6 $\mathrm{mg} / \mathrm{kg}$ ) combined with urosodeoxycholic acid (UDCA) 600 $\mathrm{mg} /$ day. However, the Grade 3 elevation of ALT persisted even after 3 weeks. Therefore, we performed steroid halfpulse therapy (mPSL $500 \mathrm{mg} /$ day, intravenously) on Day 31 after hospitalization. After that, the ALT level recovered to Grade 2 severity, so oral mPSL $(0.6 \mathrm{mg} / \mathrm{kg})$ was restarted. The steroid was tapered by $5 \mathrm{mg}$ weekly. The ALT level recovered almost to normal levels by four months after the withdrawal of nivolumab, so we stopped the oral administration of mPSL. However, the ALT level did not completely recover to the normal range after five months.

\section{Discussion}

The emergence of immune checkpoint inhibitors has drastically affected the treatment of various cancers, especially malignant melanoma. Nivolumab, an immune checkpoint inhibitor with the anti-PD-1 antibody, has recently become widely used as a new cancer therapeutic agent that enhances antitumor immunity (1). However, specific irAEs associated with immune checkpoint inhibitors have been reported (3), and little is known regarding these AEs, including hepatitis, which is reported to occur in $1.0-3.0 \%$ of nivolumab-treated patients $(4,5)$.

Mild-to-moderate hepatitis is not uncommon during nivolumab therapy but is usually self-limited. Severe hepati-
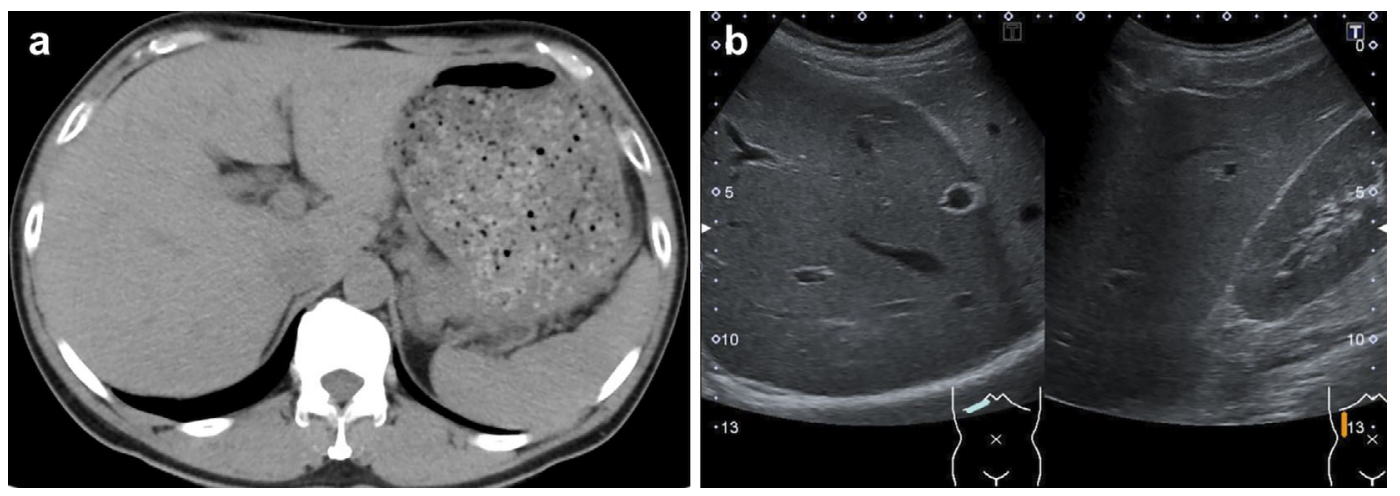

Figure 1. Computed tomography and ultrasonography findings on admission. (a) No mass in the liver. (b) No hepato-renal echo contrast. 


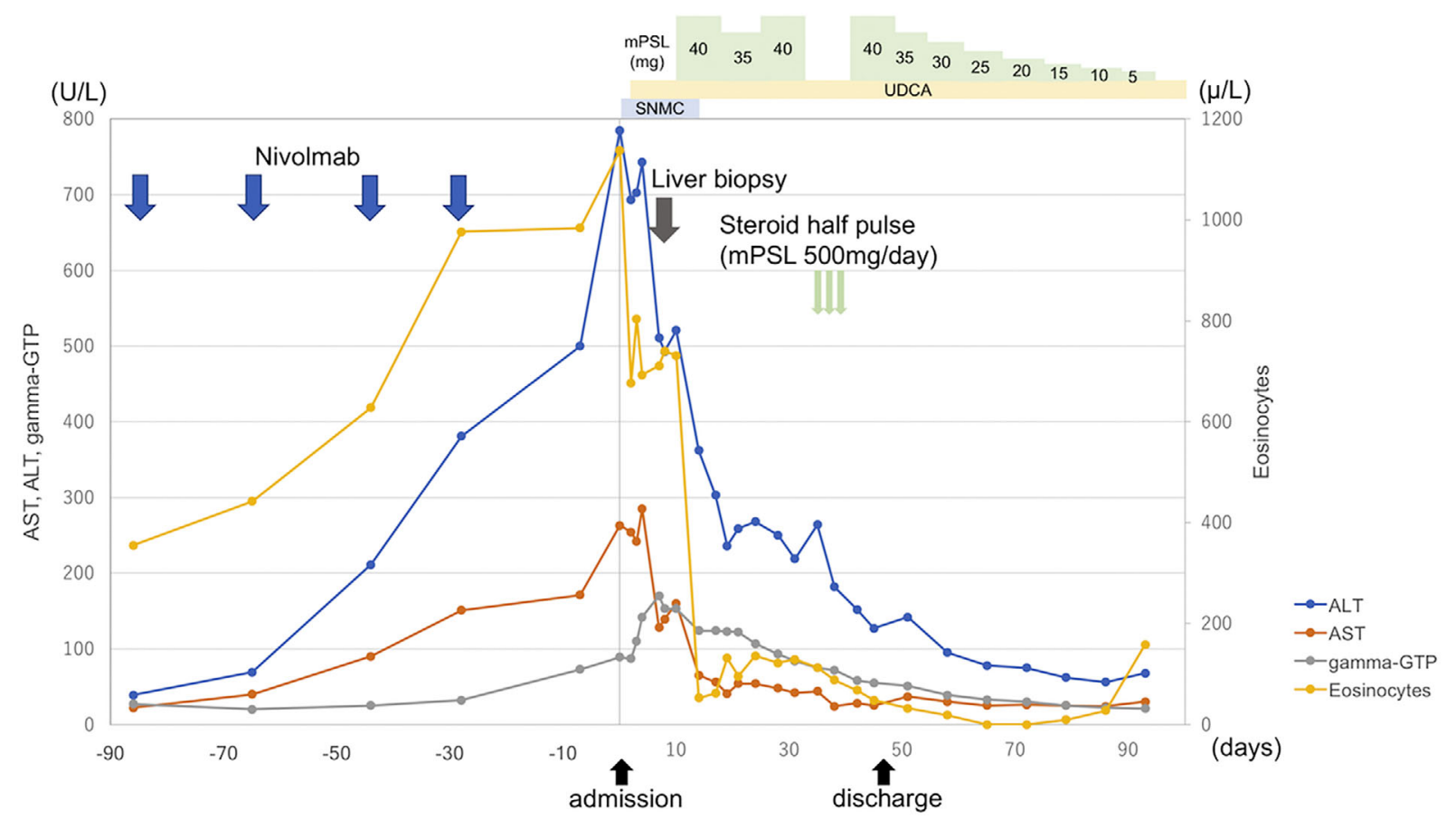

Figure 2. The time courses of laboratory data and treatment. ALT: alanine aminotransferase, AST: aspartate aminotransferase, gamma-GTP: gamma-glutamyltranspeptidase, mPSL: methylprednisolone, UDCA: ursodeoxycholic acid, SNMC: stronger neo-minophagen C
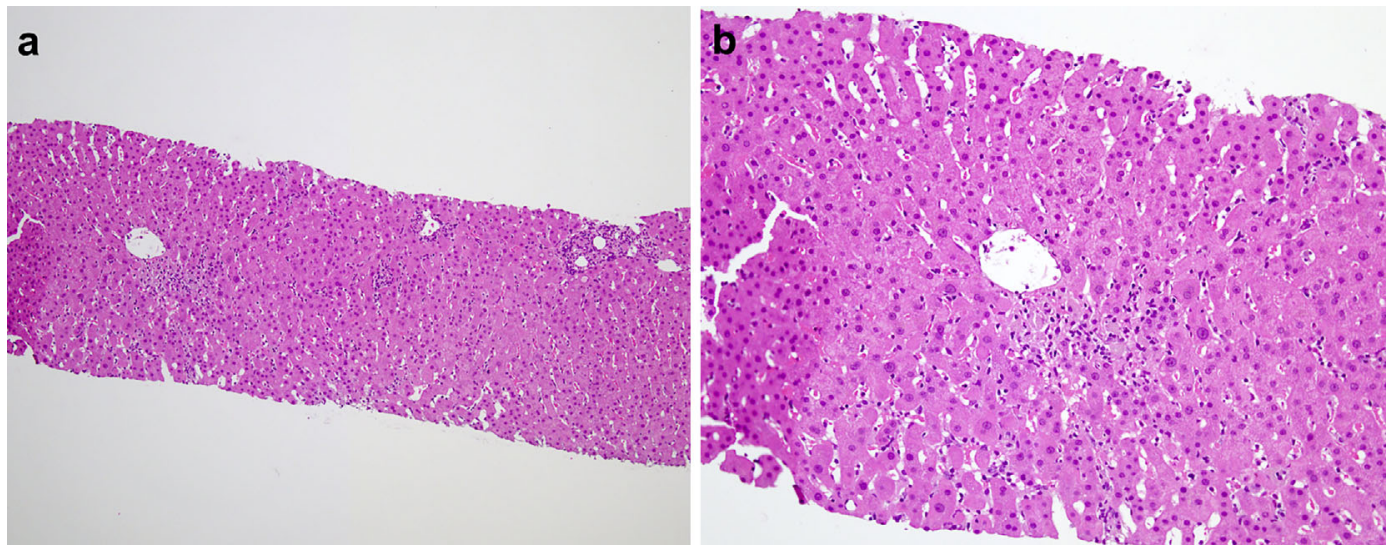

Figure 3. The pathological findings in the liver. a: Scattered necrotic foci accompanied by a small number of macrophages, lymphocytes and eosinophils were observed in the centrilobular zone. b: The portal area was inflamed with a moderate number of inflammatory cells, including lymphocytes and eosinophils.

tis occurs clinically in $0.5 \%$ to $1.5 \%$ of these patients (6-10). Hepatitis is mostly asymptomatic and detected on routine blood tests. Consequently, impaired self-tolerance from loss of T-cell inhibition induces the development of irAEs. The disadvantage of immunopotentiators is the autoimmune-related inflammation of normal tissues. These irAEs are generally manageable by immune-modulatory medications, such as steroids, but some cases can be fatal (11-14). Therefore, recognizing the timing of the onset of irAEs and their appropriate management is important. In a pooled analysis of nivolumab-treated patients, the onset of hepatitis was found to occur between 6 and 14 weeks after treatment (15). It has also been reported that the onset of liver toxicity was usually after 2 to 6 cycles, followed by the initiation of treatment after 1 to 3 months (16). However, the present case was not typical with regard to the onset of liver toxicity after treatment. Furthermore, the time course of severe hepatitis due to nivolumab therapy has hardly been reported. Accordingly, we herein report that liver injury was sustained for more than 5 months after discontinuing nivolumab therapy.

The patterns of liver injury due to immune checkpoint inhibitors are varied, ranging from hepatocellular damage to prevalent cholangitic type. Nivolumab-related liver injury usually presents as hepatocellular damage, but nivolumabinduced cholangitis has recently been reported (4). The his- 
tological characteristics of cholangitis demonstrated T-cell infiltration around the Glisson's capsule with cluster of differentiation 8 (CD8)-positive T-cells predominantly found in the intrahepatic biliary tract. The present case had a pattern of hepatocellular damage, and liver histology revealed acute hepatitis with scattered necrotic foci accompanied by a small number of macrophages, lymphocytes and eosinophils in the centrilobular zone and a moderate number of inflammatory cells, including lymphocytes and eosinophils, in the portal area, which was compatible with drug-induced liver damage without autoimmune-mediated hepatitis. In the present case, a histological examination revealed slight interface hepatitis and no cholestasis. In addition, the gamma-globulin values were normal, and all autoantibodies were negative. These findings from the clinical and pathological examinations were compatible with drug-induced hepatitis.

Nivolumab-related liver injury is likely to occur via an autoimmune-mediated mechanism, and most of cases have responded to corticosteroid or immunosuppressive therapy. The standard algorithm for the management of patients receiving nivolumab recommends monitoring the liver enzymes and initiating therapy with high doses of intravenous mPSL for patients who develop serum aminotransferase elevations above 5 times the upperlimit of normal (ULN), followed by tapering with oral mPSL (1-2 mg/kg) for at least 30 days (http://www.cancernetwork.com). If the hepatitis does not respond to steroids, mycophenylate moferil should be introduced at 500-1,000 mg, twice daily. Because the present case histologically resembled drug-induced hepatitis, our patient might have shown a poorer response to steroids and more sustained liver injury than would have been observed with autoimmune-mediated hepatitis induced by nivolumab as an irAE. In addition, the patient's liver injury may have been sustained because of the delay in starting the treatment.

$\mathrm{Li}$ et al. reported that tumor response continued to be complete remission for 14 months despite discontinuing nivolumab due to the presence of Grade 3 pneumonitis (17). Just as with our case, the remission of metastatic lung tumor was sustained, which is compatible with the continuing immune-mediated efficacy of nivolumab. Our patient also continued to show remission of his metastatic lung tumor for five months despite discontinuing nivolumab due to the presence of Grade 4 hepatitis.

In summary, we herein described a rare case of sustained liver injury after nivolumab treatment. Further studies are warranted to verify the efficacy and irAEs of nivolumab treatment in patients with various cancers.

The authors state that they have no Conflict of Interest (COI).

\section{References}

1. Pardoll DM. The blockade of immune checkpoints in cancer immunotherapy. Nat Rev Cancer 12: 252-264, 2012.

2. Guo L, Zhang H, Chen B. Nivolumab as programmed death-1 (PD-1) inhibitor for targeted immunotherapy in tumor. J Cancer $\mathbf{8}$ : 410-416, 2017.

3. Postow MA, Chesney J, Pavlick AC, et al. Nivolumab and ipilimumab versus ipilimumab in untreated melanoma. $\mathrm{N}$ Engl $\mathrm{J}$ Med 372: 2006-2017, 2015.

4. Kawakami H, Tanizaki J, Tanaka K, et al. Imaging and clinicopathological features of nivolumab-related cholangitis in patients with non-small cell lung cancer. Invest New Drugs 35: 529-536, 2017.

5. Gelsomino F, Vitale G, D’Errico A, Bertuzzi C, Andreone P, Ardizzoni A. Nivolumab-induced cholangitic liver disease: a novel form of serious liver injury. Ann Oncol 28: 671-672, 2017.

6. Topalian SL, Hodi FS, Brahmer JR, et al. Safety, activity, and immune correlates of anti-PD-1 antibody in cancer. N Engl J Med 366: 2443-2454, 2012.

7. Teply BA, Lipson EJ. Identification and management of toxicities from immune checkpoint-blocking drugs. Oncology (Williston Park) 28 (Suppl): 30-38, 2014.

8. Motzer RJ, Rini BI, McDermott DF, et al. Nivolumab for metastatic renal cell carcinoma: results of a randomized phase II trial. J Clin Oncol 33: 1430-1437, 2015.

9. Rizvi NA, Mazieres J, Planchard D, et al. Activity and safety of nivolumab, an anti-PD-1 immune checkpoint inhibitor, for patients with advanced, refractory squamous non-small-cell lung cancer (CheckMate 063): a phase 2, single-arm trial. Lancet Oncol 16: 257-265, 2015.

10. Brahmer J, Reckamp KL, Baas $P$, et al. Nivolumab versus docetaxel in advanced squamous-cell non-small-cell lung cancer. $\mathrm{N}$ Engl J Med 373: 123-135, 2015.

11. Hodi FS, O'Day SJ, McDermott DF, et al. Improved survival with ipilimumab in patients with metastatic melanoma. $\mathrm{N}$ Engl $\mathrm{J}$ Med 363: 711-723, 2010

12. Kwon ED, Drake CG, Scher HI, et al. Ipilimumab versus placebo after radiotherapy in patients with metastatic castration-resistant prostate cancer that had progressed after docetaxel chemotherapy (CA184-043): a multicentre, randomised, double-blind, phase 3 trial. Lancet Oncol 15: 700-712, 2014.

13. Eggermont AM, Chiarion-Sileni V, Grob JJ, et al. Adjuvant ipilimumab versus placebo after complete resection of high-risk stage III melanoma (EORTC 18071): a randomised, double-blind, phase 3 trial. Lancet Oncol 16: 522-530, 2015.

14. Robert C, Schachter J, Long GV, et al. Pembrolizumab versus ipilimumab in advanced melanoma. N Engl J Med 372: 25212532, 2015.

15. Weber JS, Kahler KC, Hauschild A. Management of immunerelated adverse events and kinetics of response with ipilimumab. $\mathrm{J}$ Clin Oncol 30: 2691-2697, 2012.

16. Abdel-Rahman O, ElHalawani H, Fouad M. Risk of elevated transaminases in cancer patients treated with immune checkpoint inhibitors: a meta-analysis. Expert Opin Drug Saf 14: 1507-1518, 2015.

17. Li H, Ma W, Yoneda KY, et al. Severe nivolumab-induced pneumonitis preceding durable clinical remission in a patient with refractory, metastatic lung squamous cell cancer: a case report. J Hematol Oncol 10: 64, 2017.

The Internal Medicine is an Open Access article distributed under the Creative Commons Attribution-NonCommercial-NoDerivatives 4.0 International License. To view the details of this license, please visit (https://creativecommons.org/licenses/ by-nc-nd/4.0/).

(C) 2018 The Japanese Society of Internal Medicine

Intern Med 57: 1789-1792, 2018 